\title{
First Principles Derivation of Effective Ginzburg-Landau Free Energy Models for Crystalline Systems
}

\author{
James F. Lutsko \\ Center for Nonlinear Phenomena and Complex Systems, \\ Université Libre de Bruxelles, C.P. 231, Blvd. du Triomphe, 1050 Brussels, Belgium
}

(Dated: April 17, 2018)

\begin{abstract}
The expression of the free energy density of a classical crystalline system as a gradient expansion in terms of a set of order parameters is developed using classical density functional theory. The goal here is to extend and complete an earlier derivation by Löwen et al (Europhys. Lett.9, 791, 1989). The limitations of the resulting expressions are also discussed including the boundary conditions needed for finite systems and the fact that the results cannot, at present, be used to take into account elastic relaxation.

PACS numbers: 05.20.-y,68.08.-p,65.40.Gr
\end{abstract}

\section{INTRODUCTION}

Classical Density Functional Theory (DFT) can be used to determine the thermodynamic and structural properties of classical systems based on only the interaction potential of the atoms and the external fields (including those of the walls if any) they are subject to[1]. It is based on the fact that the Helmholtz free energy is a unique functional of the local density and the goal of practical formulations of DFT is to provide an approximate form for that functional relation that can be used to calculate the properties of real systems under a variety of thermodynamic and geometric conditions[1],[2]. For inhomogeneous systems, containing e.g. surfaces or bulk interfaces, even the simplest approximate functionals can be analytically complex to work with so that from the earliest days of the development of DFT in the 1970's it has been common in such circumstances to use gradient expansions in the order parameters in order to obtain simplified forms of the theory[1]. In fact, two types of quite different gradient expansions are currently in use. The first, described by Evans[1] and refined by Haymet and Oxtoby[3],[4], yields equations for the local density which vary with the microscopic structure of the system: for example, when applied to a solid, that some terms in the equations will exhibit variations on the length scale of the lattice parameter. The second type of gradient expansion is that of Löwen, Beier and Wagner $[5,6]$ which yields a typical Ginzburg-Landau model free energy. While based on similar ideas to the first method, this results in a true long-wavelength approximation in which microscopic details, such as the underlying lattice when applied to a solid, are subsumed into the various coefficients of the gradient expansion. The resulting simplified free energy functional therefore takes the form of a typical phenomenological Landau theory, but with coefficients that can be calculated from microscopic information. This theory has been used to discuss surface melting, the nucleation of freezing in a bulk fluid[7],[8] and solid-solid phase transitions[9].

The purpose of this paper is twofold. The first goal is to review the two types of gradient expansions in order to clarify the differences between them. The need to do so is motivated by certain criticisms that have been made in the literature[10] about the inadequacy of the gradient expansions: as shown below, these seem likely to be relevant to the older class but not to the Ginzburg-Landau model. The second goal is to clarify, extend and complete the derivation of the Ginzburg-Landau models. The derivation currently in the literature[5],[6] was developed only for planar interfaces and is also incomplete. Furthermore, issues such as the appropriate boundary conditions for finite systems have not been addressed.

In the next Section, the required elements of classical DFT are reviewed and the older form of the gradient expansion is discussed. Section III presents in detail a derivation of the Ginzburg-Landau model of Löwen et al. Finally, in the last Section the differences between the two types of expansion are discussed, as are the advantages and limitations of the models.

\section{DENSITY FUNCTIONAL THEORY}

Consider a system of $N$ atoms with positions and momenta $\vec{q}_{i}$ and $\vec{p}_{i}$ respectively and mass $m_{i}$. The atoms interact via a pair potential $v(\vec{r})$ and are also subject to the effect of an external field $\phi(\vec{r})$ which includes any one-body forces such as gravity or applied electrical fields as well as the effects of any walls. The local number density 


$$
\widehat{\rho}(\vec{r})=\sum_{i=1}^{N} \delta\left(\vec{r}-\vec{q}_{i}\right)
$$

and its average in a grand-canonical ensemble is denoted

$$
\rho(\vec{r})=\langle\widehat{\rho}(\vec{r})\rangle .
$$

The grand distribution is

$$
f=\Xi^{-1} \frac{1}{N ! h^{3 N}} \exp (-\beta H+\beta \mu N)
$$

where $h$ is Planck's constant, $\beta=1 / k_{B} T$ is the inverse temperature, $\mu$ is the chemical potential and the Hamiltonian is

$$
H=\sum_{i=1}^{N} \frac{1}{2 m_{i}} p_{i}^{2}+\sum_{i<j} v\left(\vec{q}_{i j}\right)+\int d \vec{r} \widehat{\rho}(\vec{r}) \phi(\vec{r})
$$

The grand partition function is

$$
\Xi=\sum_{N} \int d \vec{q}^{N} d \vec{p}^{N} \frac{1}{N ! h^{3 N}} \exp (-\beta H+\mu N)
$$

and it is related to the grand potential, $\Omega$, as

$$
\Omega=-k_{B} T \ln \Xi
$$

The key results of DFT[1],[2] are that (a) there is a one-to-one correspondence between fields $\phi(\vec{r})$ and average density distributions $\rho(\vec{r})$ leading to the fact that (b) there exists a unique functional of the density $F[\rho]$ with the property that the functional

$$
\Omega([n],[\phi]) \equiv F[n]-\mu \int d \vec{r} n(\vec{r})+\int d \vec{r} n(\vec{r}) \phi(\vec{r})
$$

is minimized, for a fixed field, by the equilibrium density

$$
\left.\frac{\delta \Omega}{\delta n}\right|_{\phi, \mu, T}(n=\rho)=0
$$

where the notation indicates that the variation is performed at fixed external field $\phi$ as well as fixed chemical potential and temperature[1],[2]. The functional $F[n]$ is commonly written as $F[n]=F_{i d}[n]+F_{e x}[n]$ with the ideal contribution given by

$$
\beta F_{i d}[n]=\int d \vec{r}\left(n(\vec{r}) \ln \left(\Lambda^{3} n(\vec{r})\right)-n(\vec{r})\right)
$$

and where the excess contribution is in general unknown. However, it is related to the $m$-body direct correlation functions by

$$
c_{n}\left(\vec{r}_{1}, \ldots, \vec{r}_{m}\right)=-\frac{\delta^{m} \beta F_{e x}[n]}{\delta n\left(\vec{r}_{1}\right) \ldots \delta n\left(\vec{r}_{m}\right)} .
$$

Combining eqs.(7)-(10), the Euler-Lagrange equation can be written as

$$
\ln \left(\Lambda^{3} n(\vec{r})\right)-c_{1}(\vec{r})-\beta \mu+\beta \phi(\vec{r})=0 .
$$

Finally, in terms of these quantities, the Helmholtz free energy $A$ is

$$
A=F[\rho]+\int d \vec{r} \rho(\vec{r}) \phi(\vec{r})
$$


All practical uses of DFT are based on approximate functionals $F_{e x}[n]$ motivated by various physical reasons. Given such an approximation, one then has two choices as to how to proceed. The first is to use the functional to derive the one-body direct correlation function from eq.(10) and then to derive the density from eq.(11). (Note that in bulk systems, the only external force of interest is often that associated with the walls which contain the system. As this volume goes to infinity, it is expected that interior points are unaffected by the wall-force so that in these so-called "self-sustaining" systems, one can take $\beta \phi(\vec{r})=0$.) The second approach is to parameterize the density and to then determine the parameters via eq(8). To be specific, let the parameterized density be $n(\vec{r})=\rho(\vec{r} ; \Gamma)$ where $\Gamma=\left\{\Gamma_{a}\right\}$ represents a family of parameters. In a bulk system, the parameters are constants and eq.(8) implies that

$$
\frac{\partial F}{\partial \Gamma_{a}}-\mu \frac{\partial N}{\partial \Gamma_{a}}+\int d \vec{r} \frac{\partial \rho(\vec{r} ; \Gamma)}{\partial \Gamma_{a}} \phi(\vec{r})=0
$$

If the parameters depended on position, the result would be

$$
\left[\frac{\delta F}{\delta n(\vec{r})}\right]_{\rho(\vec{r} ; \Gamma)} \frac{\partial \rho(\vec{r} ; \Gamma)}{\partial \Gamma_{a}(\vec{r})}-\mu \frac{\partial \rho(\vec{r} ; \Gamma)}{\partial \Gamma_{a}(\vec{r})}+\phi(\vec{r}) \frac{\partial \rho(\vec{r} ; \Gamma)}{\partial \Gamma_{a}(\vec{r})}=0 .
$$

One trivial example of such a parameterization is a bulk liquid in which case one takes $\rho(\vec{r} ; \Gamma)=\rho_{0}$ for some constant $\rho_{0}$. Then, since the field is taken to be zero in the interior, the Euler-Lagrange equations simply give the usual thermodynamic relation

$$
\frac{\partial F}{\partial \rho_{0}}-\mu V=0
$$

A less trivial example is that of a bulk crystalline solid which is typically parameterized by a sum of Gaussians giving

$$
\rho(\vec{r} ; \Gamma)=\sum_{i=1}^{N_{V}}\left(\frac{\alpha}{\pi}\right)^{3 / 2} \exp \left(-\alpha\left(\vec{r}-\vec{R}_{i}\right)^{2}\right)
$$

where $\left\{R_{i}\left(\rho_{0}\right)\right\}_{i=1}^{N_{v}}$ are the Bravais lattice vectors within the volume $V$ and are, as indicated, functions of the average density $\rho_{0}$. Then, the parameters are $\Gamma=\left\{\alpha, \rho_{0}\right\}$ and since the field is taken to be zero in the interior, the EulerLagrange equations reduce to

$$
\begin{aligned}
\frac{\partial F}{\partial \alpha} & =0 \\
\frac{\partial F}{\partial \rho_{0}}-\mu V & =0 .
\end{aligned}
$$

A solid-liquid interfacial system could be described by taking, e.g., $\alpha$ to be spatially dependent and by using eq.(14). Note that this requires that the free energy functional $F[n]$ be known not only for bulk systems but for inhomogeneous systems as well. Given this functional, the choice of whether to use a parameterized density or to directly solve eq.(11) is a matter of convenience.

\section{A. Gradient Expansion}

The classical gradient expansion of DFT was described by Evans[1] and given a more modern derivation by Haymet and Oxtoby[3],[4]. Here, the latter derivation is reviewed so as to contrast with the Ginzburg-Landau theory discussed below.

Equation(10) can be functionally integrated in density space. Define a parameterized $n_{\lambda}(\vec{r})=\bar{n}_{0}+$ $\lambda\left(n_{1}(\vec{r})-\bar{n}_{0}\right)$ where $n_{1}(\vec{r})=\rho(\vec{r} ; \Gamma(\vec{r}))$ is the state of interest and $\bar{n}_{0}$ corresponds to any convenient uniform (i.e. liquid) state.Then two integrations and adding and subtracting the same expression for $n_{1}(\vec{r})=\bar{n}_{1}$ gives

$$
\begin{aligned}
\beta F_{e x}\left[n_{1}\right]-\beta F_{e x}\left(\bar{n}_{1}\right)= & -\int d \vec{r}_{1} d \vec{r}_{2} \int_{0}^{1} \int_{0}^{\lambda} c_{2}\left(\vec{r}_{1}, \vec{r}_{2} ; n_{\lambda^{\prime}}\right)\left(n_{1}\left(\vec{r}_{1}\right)-\bar{n}_{0}\right)\left(n_{1}\left(\vec{r}_{2}\right)-\bar{n}_{0}\right) d \lambda d \lambda^{\prime} . \\
& +\int d \vec{r}_{1} d \vec{r}_{2} \int_{0}^{1} \int_{0}^{\lambda} c_{2}\left(\vec{r}_{1}, \vec{r}_{2} ; \bar{n}_{\lambda^{\prime}}\right)\left(\bar{n}_{1}-\bar{n}_{0}\right)\left(\bar{n}_{1}-\bar{n}_{0}\right) d \lambda d \lambda^{\prime} .
\end{aligned}
$$


Adding in the ideal contribution gives

$$
\begin{aligned}
\beta F\left[n_{1}\right]-\beta F\left(\bar{n}_{1}\right)= & \int d \vec{r}_{1}\left(n_{1}\left(\vec{r}_{1}\right) \ln n_{1}\left(\vec{r}_{1}\right)-\bar{n}_{1} \ln \bar{n}_{1}\right) \\
& -\int d \vec{r}_{1} d \vec{r}_{2} \int_{0}^{1} \int_{0}^{\lambda} c_{2}\left(\vec{r}_{1}, \vec{r}_{2} ; n_{\lambda^{\prime}}\right)\left(n_{1}\left(\vec{r}_{1}\right)-\bar{n}_{0}\right)\left(n_{1}\left(\vec{r}_{2}\right)-\bar{n}_{0}\right) d \lambda d \lambda^{\prime} . \\
& +\int d \vec{r}_{1} d \vec{r}_{2} \int_{0}^{1} \int_{0}^{\lambda} c_{2}\left(\vec{r}_{1}, \vec{r}_{2} ; \bar{n}_{\lambda^{\prime}}\right)\left(\bar{n}_{1}-\bar{n}_{0}\right)\left(\bar{n}_{1}-\bar{n}_{0}\right) d \lambda d \lambda^{\prime},
\end{aligned}
$$

and it should be noted that all of these relations are exact.

Haymet and Oxtoby then introduce the following approximations. First, the two-body dcf is expanded about that of a uniform system as $c_{2}\left(\vec{r}_{1}, \vec{r}_{2} ; n_{\lambda^{\prime}}\right)=c_{2}\left(r_{12} ; n_{0}\right)+\ldots$ and higher order terms dropped giving

$$
\begin{aligned}
\beta F[n]-\beta F\left(\bar{n}_{1}\right) \simeq & \int d \vec{r}_{1}\left(n_{1}\left(\vec{r}_{1}\right) \ln n_{1}\left(\vec{r}_{1}\right)-\bar{n}_{1} \ln \bar{n}_{1}\right) \\
& -\frac{1}{2} \int c_{2}\left(r_{12} ; n_{0}\right)\left(n_{1}\left(\vec{r}_{1}\right)-\bar{n}_{1}\right)\left(n_{1}\left(\vec{r}_{2}\right)+\bar{n}_{1}\right) d \vec{r}_{1} d \vec{r}_{2}
\end{aligned}
$$

Introducing the local free energy difference for a uniform system

$$
\begin{aligned}
\Delta \beta f\left(\vec{r}_{1} ; \Gamma_{0}\right)= & \rho_{1}\left(\vec{r}_{1} ; \Gamma_{0}\right) \ln \rho_{1}\left(\vec{r}_{1} ; \Gamma_{0}\right)-\bar{\rho}_{1} \ln \bar{\rho}_{1} \\
& -\frac{1}{2} \int c_{2}\left(r_{12} ; n_{0}\right)\left(\rho_{1}\left(\vec{r}_{1} ; \Gamma_{0}\right)+\bar{\rho}_{1}\right)\left(\rho_{1}\left(\vec{r}_{2} ; \Gamma_{0}\right)-\bar{\rho}_{1}\right) d \vec{r}_{2}
\end{aligned}
$$

the last result can be written more suggestively as

$$
\begin{aligned}
\beta F[n]-\beta F\left(\bar{n}_{1}\right) \simeq & \int d \vec{r}_{1} \Delta \beta f\left(\vec{r}_{1} ; \Gamma\left(\vec{r}_{1}\right)\right) \\
& -\frac{1}{2} \int c_{2}\left(r_{12} ; n_{0}\right)\left(\rho_{1}\left(\vec{r}_{1} ; \Gamma_{0}\right)+\bar{\rho}_{1}\right)\left(\rho_{1}\left(\vec{r}_{2} ; \Gamma_{0}\right)-\bar{\rho}_{1}\right) d \vec{r}_{1} d \vec{r}_{2}
\end{aligned}
$$

so that the first term on the right has the form of a mean-field approximation. The second term can be expanded to give, up to second order in gradients of the order parameter

$$
\begin{aligned}
& \beta F[n]-\beta F\left(\bar{n}_{1}\right) \simeq \int d \vec{r}_{1} \Delta \beta f\left(\vec{r}_{1} ; \Gamma\left(\vec{r}_{1}\right)\right) \\
& -\frac{1}{2} \int c_{2}\left(r_{12} ; n_{0}\right)\left(\rho_{1}\left(\vec{r}_{1} ; \Gamma\left(\vec{r}_{1}\right)\right)+\bar{\rho}_{1}\right) \frac{\partial \rho_{1}\left(\vec{r}_{2} ; \Gamma\left(\vec{r}_{1}\right)\right)}{\partial \Gamma\left(\vec{r}_{1}\right)} \vec{r}_{21} \cdot \vec{\nabla} \Gamma\left(\vec{r}_{1}\right) d \vec{r}_{1} d \vec{r}_{2} \\
& -\frac{1}{4} \int c_{2}\left(r_{12} ; n_{0}\right)\left(\rho_{1}\left(\vec{r}_{1} ; \Gamma\left(\vec{r}_{1}\right)\right)+\bar{\rho}_{1}\right) \frac{\partial \rho_{1}\left(\vec{r}_{2} ; \Gamma\left(\vec{r}_{1}\right)\right)}{\partial \Gamma\left(\vec{r}_{1}\right)} \vec{r}_{21} \vec{r}_{21} \cdot \vec{\nabla} \vec{\nabla} \Gamma\left(\vec{r}_{1}\right) d \vec{r}_{1} d \vec{r}_{2} \\
& -\frac{1}{4} \int c_{2}\left(r_{12} ; n_{0}\right)\left(\rho_{1}\left(\vec{r}_{1} ; \Gamma\left(\vec{r}_{1}\right)\right)+\bar{\rho}_{1}\right) \frac{\partial_{1}^{2} \rho\left(\vec{r}_{2} ; \Gamma\left(\vec{r}_{1}\right)\right)}{\partial \Gamma^{2}\left(\vec{r}_{1}\right)}\left(\vec{r}_{21} \cdot \vec{\nabla} \Gamma\left(\vec{r}_{1}\right)\right)^{2} d \vec{r}_{1} d \vec{r}_{2}
\end{aligned}
$$

$\mathrm{OH}$ use a specific parameterization

$$
\rho_{1}(\vec{r} ; \Gamma(\vec{r}))=\rho_{0}\left(1+\Gamma_{0}(\vec{r})+\sum_{n>0} \exp \left(i \vec{K}_{n} \cdot \vec{r}\right) \Gamma_{n}(\vec{r})\right)
$$

where $\vec{K}_{n}$ is the $n t h$ reciprocal lattice vector. Substituting into the expression for the free energy gives

$$
\begin{aligned}
\beta F\left[n_{1}\right]-\beta F\left(\bar{n}_{1}\right) \simeq & \int d \vec{r}_{1} \Delta \beta f\left(\vec{r}_{1} ; \Gamma\left(\vec{r}_{1}\right)\right) \\
& -\frac{1}{2} \rho_{0} \int c_{2}\left(r_{12} ; \rho_{0}\right)\left(\rho_{0}+n_{0} \Gamma_{0}\left(\vec{r}_{1}\right)+\bar{n}_{1}\right) \vec{r}_{21} \cdot \vec{\nabla}_{\Gamma_{0}}\left(\vec{r}_{1}\right) d \vec{r}_{1} d \vec{r}_{2} \\
& -\frac{1}{2} \rho_{0} \sum_{n>0} \int c_{2}\left(r_{12} ; \rho_{0}\right) \exp \left(i \vec{K}_{n} \cdot \vec{r}_{12}\right) \Gamma_{n}\left(\vec{r}_{1}\right) \vec{r}_{21} \cdot \vec{\nabla} \Gamma_{n}\left(\vec{r}_{1}\right) d \vec{r}_{1} d \vec{r}_{2} \\
& -\frac{1}{4} \rho_{0} \int c_{2}\left(r_{12} ; \rho_{0}\right)\left(\rho_{0}+\rho_{0} \Gamma_{0}\left(\vec{r}_{1}\right)+\bar{\rho}_{1}\right) \vec{r}_{21} \vec{r}_{21} \cdot \vec{\nabla} \vec{\nabla} \Gamma_{0}\left(\vec{r}_{1}\right) d \vec{r}_{1} d \vec{r}_{2} \\
& -\frac{1}{4} \rho_{0} \sum_{n>0} \int c_{2}\left(r_{12} ; \rho_{0}\right) \exp \left(i \vec{K}_{n} \cdot \vec{r}_{12}\right) \Gamma_{n}\left(\vec{r}_{1}\right) \vec{r}_{21} \vec{r}_{21} \cdot \vec{\nabla} \vec{\nabla}_{n}\left(\vec{r}_{1}\right) d \vec{r}_{1} d \vec{r}_{2} .
\end{aligned}
$$


The linear terms vanish by symmetry of the uniform fluid and, after changing variables and integrating by parts, the final result is

$$
\begin{aligned}
\beta F\left[n_{1}\right]-\beta F\left(\bar{n}_{1}\right) \simeq & \int d \vec{r}_{1} \Delta \beta f\left(\vec{r}_{1} ; \Gamma\left(\vec{r}_{1}\right)\right) \\
& +\frac{1}{4} \rho_{0}^{2} \int c_{2}\left(r_{12} ; \rho_{0}\right)\left(\vec{r}_{21} \cdot \Gamma_{0}\left(\vec{r}_{1}\right)\right)^{2} d \vec{r}_{1} d \vec{r}_{12} \\
& +\frac{1}{4} \rho_{0}^{2} \sum_{n>0} \int c_{2}\left(r_{12} ; \rho_{0}\right) \exp \left(i \vec{K}_{n} \cdot \vec{r}_{12}\right)\left(\vec{r}_{21} \cdot \vec{\nabla}_{n}\left(\vec{r}_{1}\right)\right)^{2} d \vec{r}_{1} d \vec{r}_{12} .
\end{aligned}
$$

This can be written in the form

$$
\beta F\left[n_{1}\right]-\beta F\left(\bar{n}_{1}\right) \simeq \int d \vec{r}\left[\Delta \beta f(\vec{r} ; \Gamma(\vec{r}))+\frac{1}{2} \sum_{a b} K_{i j}^{a b}\left(\partial_{i} \Gamma_{a}(\vec{r})\right)\left(\partial_{j} \Gamma_{b}(\vec{r})\right)\right]
$$

with

$$
K_{i j}^{a b}=\delta_{a b} \frac{1}{2} \rho_{0}^{2} \int \exp \left(i \vec{K}_{a} \cdot \vec{r}\right) r_{i} r_{j} c_{2}\left(r ; n_{0}\right) d \vec{r}
$$

The main criticisms of this theory are that (a) all correlation functions are eventually expressed in terms of an expansion about the uniform liquid and these expansions truncated at the two-body dcf; (b) the latter stages of the derivation depend on a particular parameterization of the density and (c) the mean field contribution, $\Delta f\left(\vec{r}_{1} ; \Gamma\left(\vec{r}_{1}\right)\right)$, is not a truly long-wavelength approximation as it clearly exhibits variations on the length scale of the lattice. To clarify the last point: in a long-wavelength theory, one would expect to see the term

$$
\int d \vec{r}_{1} \Delta \beta f\left(\vec{r}_{1} ; \Gamma\left(\vec{r}_{1}\right)\right)
$$

replaced by something like

$$
\int d \vec{R} \int d \vec{r}_{1} \Delta \beta f\left(\vec{r}_{1} ; \Gamma(\vec{R})\right)=\int d \vec{R} \Delta \beta F(\Gamma(\vec{R}))
$$

so that the variational equations for the order parameters do not contain terms that vary over microscopic length scales.

\section{EFFECTIVE GINZBURG-LANDAU MODEL}

Ideally, one would like to obtain a Helmholtz free energy in which microscopic length scales do not occur of the generic form

$$
\beta F[\rho]=\int d \vec{r}\left[f_{0}(\Gamma(\vec{r}))+\frac{1}{2} K_{i j}^{a b}(\Gamma(\vec{r}))\left(\frac{\partial}{\partial r_{i}} \Gamma_{a}(\vec{r})\right)\left(\frac{\partial}{\partial r_{j}} \Gamma_{b}(\vec{r})\right)+\ldots\right]
$$

in which dependence on the atomic structure has been "integrated out". Furthermore, one would expect the first term to take the typical mean-field form of the bulk free energy evaluated for the local order parameters,

$$
V f_{0}\left(\Gamma\left(\vec{r}_{1}\right)\right)=\left.\beta F[\rho]\right|_{\Gamma=\Gamma\left(\vec{r}_{1}\right)} \equiv \beta F_{b u l k}(\Gamma(\vec{r}))
$$

where $\beta \Phi_{\text {bulk }}\left(\Gamma_{0}\right)$ is the free energy of a bulk system with spatially uniform order parameters $\Gamma_{0}$. Given a free energy functional of this form, and truncating at second order in the gradients, the Euler-Lagrange equations, eqs.(13), would become

$$
\begin{gathered}
K_{i j}^{a b}(\Gamma(\vec{r})) \frac{\partial^{2}}{\partial r_{i} \partial r_{j}} \Gamma_{b}(\vec{r})+g_{i j}^{a b c}(\Gamma(\vec{r}))\left(\frac{\partial}{\partial r_{i}} \Gamma_{b}(\vec{r})\right)\left(\frac{\partial}{\partial r_{j}} \Gamma_{c}(\vec{r})\right)-\frac{\partial}{\partial \Gamma_{a}(\vec{r})} \beta F_{b u l k}(\Gamma(\vec{r}))+\mu \delta_{a 0}=0 \\
g_{i j}^{a b c}(\Gamma)=\frac{\partial}{\partial \Gamma_{c}} K_{i j}^{a b}(\Gamma)-\frac{1}{2} \frac{\partial}{\partial \Gamma_{a}} K_{i j}^{b c}(\Gamma)
\end{gathered}
$$


where it is assumed that $\Gamma_{0}$ is the average density. These variational equations are derived under the usual assumption that the values of the order parameters on the boundaries are held fixed.

The idea presented here is to first break up the volume of the systems into cells containing a single atom. Then, withing the cells, the spatial dependence of the slowly varying parameters is expressed as a gradient expansion. Since these parameters are, a priori assumed to vary little over atomic length scales, it is assumed that the gradient expansion can be truncated without serious approximation. Finally, the resulting expressions for each cell are resummed thus separating the atomic and structural length scales.

\section{A. Initial gradient expansion}

To begin, it is convenient to use the representation

$$
\beta F[\rho]=\int d \vec{r} \beta f(\vec{r} ;[\rho])
$$

where $f(\vec{r} ;[\Gamma])$ can be expressed in terms of the one-body dcf and the density using the methods of the previous Section,

$$
\beta f(\vec{r} ;[\rho])=-\int_{0}^{1} c_{1}(\vec{r} ;[\lambda \rho]) \rho(\vec{r}) d \lambda .
$$

An important assumption made throughout this Section is that the density is parameterized by a set of order parameters which might include e.g. the average density but that the underlying lattice structure, including the lattice constant, is held fixed. For example, the Haymet-Oxtoby parameterization given in eq.(24) is of this form since the reciprocal lattice vectors $\vec{K}_{n}$ are held fixed; another example is that used in ref.([7])

$$
\rho(\vec{r})=\eta_{0}(\vec{r})\left(\frac{\alpha(\vec{r})}{\pi}\right)^{3 / 2} \sum_{n} \exp \left(-\alpha(\vec{r})\left(\vec{r}-\vec{R}_{n}\right)^{2}\right)
$$

where the two order parameters are $\Gamma_{0}(\vec{r})=\eta_{0}(\vec{r})$ and $\Gamma_{1}(\vec{r})=\alpha(\vec{r})$ but the lattice vectors $\vec{R}_{n}$ are held constant. Furthermore, attention here will be restricted to the most important case of simple Bravis lattices, such as FCC and BCC, with a single atom per unit cell. Notice that this also includes as a special case the nonuniform liquid which results in both parameterizations from taking $\Gamma_{n}(\vec{r})=0$ for $n \geq 0$. These models are therefore sufficiently flexible to describe the solid-liquid interface, as well as e.g. the gas-liquid interface.

To begin, partition space into the Wigner-Seitz cells where the center of the $n$th cell, $V_{n}$, will be at $\vec{R}_{n}$. and all cells have volume $V_{w s}$. The expression for the free energy can therefore be written as

$$
\beta F[\rho]=\sum_{n=1}^{N_{V}} \int_{V_{n}} d \vec{r} \beta f(\vec{r} ;[\rho])
$$

In each cell, the density is expanded about that of a system with uniform order parameter

$$
\begin{aligned}
\beta F[\rho]= & \sum_{n=1}^{N_{V}} \int_{V_{n}} d \vec{r} \beta f\left(\vec{r} ;\left[\rho_{n}\right]\right) \\
& +\left.\sum_{n=1}^{N_{V}} \int_{V_{n}} d \vec{r} \int d \vec{r}_{1} \frac{\delta \beta f\left(\vec{r}_{2}[\rho]\right)}{\delta \rho\left(\vec{r}_{1}\right)}\right|_{\rho_{n}} \delta \rho\left(\vec{r}_{1} ; \Gamma\left(\vec{r}_{1}\right), \Gamma_{n}\right) \\
& +\left.\frac{1}{2} \sum_{n=1}^{N_{V}} \int_{V_{n}} d \vec{r} \int d \vec{r}_{1} d \vec{r}_{2} \frac{\left.\delta^{2} \beta f\left(\vec{r}_{2} ; \rho\right]\right)}{\delta \rho\left(\vec{r}_{1}\right) \delta \rho\left(\vec{r}_{2}\right)}\right|_{\rho_{n}} \delta \rho\left(\vec{r}_{1} ; \Gamma\left(\vec{r}_{1}\right), \Gamma_{n}\right) \delta \rho\left(\vec{r}_{2} ; \Gamma\left(\vec{r}_{2}\right), \Gamma_{n}\right) \\
& +\ldots
\end{aligned}
$$

where

$$
\delta \rho\left(\vec{r}_{1} ; \Gamma\left(\vec{r}_{1}\right), \Gamma_{n}\right)=\rho\left(\vec{r}_{1} ; \Gamma\left(\vec{r}_{1}\right)\right)-\rho\left(\vec{r}_{1} ; \Gamma_{n}\right)
$$


and $\Gamma_{n}=\Gamma\left(\vec{R}_{n}\right)$ is the value of order parameter at the center of the nth cell. At this point, all terms are retained and no truncation is imposed. Next, the dependence of the densities on the order parameters is expanded using

$$
\begin{aligned}
\rho\left(\vec{r}_{1} ; \Gamma\left(\vec{r}_{1}\right)\right)= & \rho\left(\vec{r}_{1} ; \Gamma_{n}\right)+\sum_{a}\left(\Gamma_{a}\left(\vec{r}_{1}\right)-\Gamma_{n, a}\right) \frac{\partial \rho\left(\vec{r}_{1} ; \Gamma_{n}\right)}{\partial \Gamma_{n, a}} \\
& +\frac{1}{2} \sum_{a b}\left(\Gamma_{a}\left(\vec{r}_{1}\right)-\Gamma_{n, a}\right)\left(\Gamma_{b}\left(\vec{r}_{1}\right)-\Gamma_{n, b}\right) \frac{\partial^{2} \rho\left(\vec{r}_{1} ; \Gamma_{n}\right)}{\partial \Gamma_{n, a} \partial \Gamma_{n, b}} \\
& +\ldots
\end{aligned}
$$

as is the spatial dependence of the order parameters themselves

$$
\Gamma_{a}\left(\vec{r}_{1}\right)=\Gamma_{a}\left(\vec{R}_{n}\right)+\left(\vec{r}_{1}-\vec{R}_{n}\right) \cdot\left(\vec{\nabla} \Gamma_{a}\right)_{n}+\frac{1}{2}\left(\vec{r}_{1}-\vec{R}_{n}\right)\left(\vec{r}_{1}-\vec{R}_{n}\right):\left(\vec{\nabla} \vec{\nabla} \Gamma_{a}\right)_{n}+\ldots
$$

The key assumption made is that terms of order $\nabla^{n} \Gamma$ may be neglected for some $n>n_{0}$. Then, the functional expansion, eq.(38), is only needed up to order $n_{0}$ as well. Taking $n_{0}=2$ and combining eqs.(37-41) gives

$$
\begin{aligned}
& \beta F[\Gamma]=\sum_{n=0}^{N_{V}} \int_{V_{n}} d \vec{r} \beta f\left(\vec{r} ;\left[\rho_{n}\right]\right) \\
& +\left.\sum_{n=0}^{N_{V}} \int_{V_{n}} d \vec{r} \int d \vec{r}_{1} \frac{\delta \beta f\left(\vec{r}_{i}[\Gamma]\right)}{\delta \rho\left(\vec{r}_{1}\right)}\right|_{\rho_{n}}\left(\vec{r}_{1}-\vec{R}_{n}\right) \cdot\left(\vec{\nabla} \Gamma_{n, a}\right) \frac{\partial \rho\left(\vec{r}_{1} ; \Gamma_{n}\right)}{\partial \Gamma_{n, a}} \\
& +\left.\frac{1}{2} \sum_{n=0}^{N_{V}} \int_{V_{n}} d \vec{r} \int d \vec{r}_{1} \frac{\delta \beta f(\vec{r} ;[\Gamma])}{\delta \rho\left(\vec{r}_{1}\right)}\right|_{\rho_{n}}\left(\vec{r}_{1}-\vec{R}_{n}\right)\left(\vec{r}_{1}-\vec{R}_{n}\right):\left(\vec{\nabla} \vec{\nabla} \Gamma_{n, a}\right) \frac{\partial \rho\left(\vec{r}_{1} ; \Gamma_{n}\right)}{\partial \Gamma_{n, a}} \\
& +\left.\frac{1}{2} \sum_{n=0}^{N_{V}} \int_{V_{n}} d \vec{r} \int d \vec{r}_{1} \frac{\delta \beta f(\vec{r} ;[\Gamma])}{\delta \rho\left(\vec{r}_{1}\right)}\right|_{\rho_{n}}\left(\vec{r}_{1}-\vec{R}_{n}\right)\left(\vec{r}_{1}-\vec{R}_{n}\right):\left(\vec{\nabla} \Gamma_{n, a}\right)\left(\vec{\nabla} \Gamma_{n, b}\right) \frac{\partial^{2} \rho\left(\vec{r}_{1} ; \Gamma_{n}\right)}{\partial \Gamma_{n, a} \partial \Gamma_{n, b}}
\end{aligned}
$$

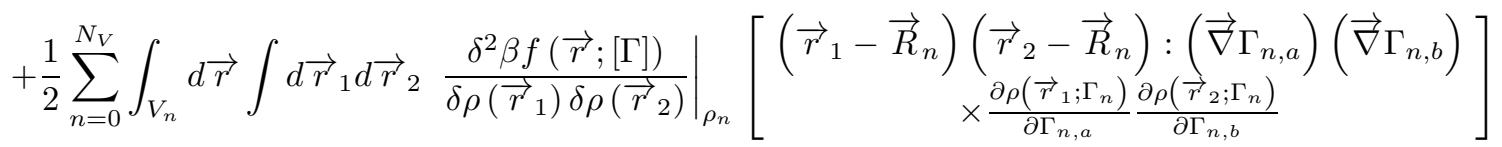

Because the terms involving functional derivatives are being evaluated at constant values of the order parameters, they exhibit the symmetry of a bulk solid. In particular, they are invariant with respect to translations by a lattice vector so a change of variables yields

$$
\begin{aligned}
\beta F[\Gamma]= & \sum_{n=0}^{N_{V}} \int_{V_{n}} d \vec{r} \beta f\left(\vec{r} ;\left[\rho_{n}\right]\right) \\
& +\left.\sum_{n=0}^{N_{V}} \int_{V_{n}} d \vec{r} \int d \vec{r}_{1} \frac{\delta \beta f(\vec{r} ;[\Gamma])}{\delta \rho\left(\vec{r}_{1}\right)}\right|_{\rho_{n}} \vec{r}_{1} \cdot\left(\vec{\nabla} \Gamma_{n, a}\right) \frac{\partial \rho\left(\vec{r}_{1} ; \Gamma_{n}\right)}{\partial \Gamma_{n, a}} \\
& +\left.\frac{1}{2} \sum_{n=0}^{N_{V}} \int_{V_{n}} d \vec{r} \int d \vec{r}_{1} \frac{\delta \beta f(\vec{r} ;[\Gamma])}{\delta \rho\left(\vec{r}_{1}\right)}\right|_{\rho_{n}} \vec{r}_{1} \vec{r}_{1} \cdot\left(\vec{\nabla} \vec{\nabla} \Gamma_{n, a}\right) \frac{\partial \rho\left(\vec{r}_{1} ; \Gamma_{n}\right)}{\partial \Gamma_{n, a}} \\
& +\left.\frac{1}{2} \sum_{n=0}^{N_{V}} \int_{V_{n}} d \vec{r} \int d \vec{r}_{1} \frac{\delta \beta f(\vec{r} ;[\Gamma])}{\delta \rho\left(\vec{r}_{1}\right)}\right|_{\rho_{n}} \vec{r}_{1} \vec{r}_{1} \cdot\left(\vec{\nabla} \Gamma_{n, a}\right)\left(\vec{\nabla} \Gamma_{n, b}\right) \frac{\partial^{2} \rho\left(\vec{r}_{1} ; \Gamma_{n}\right)}{\partial \Gamma_{n, a} \partial \Gamma_{n, b}} \\
& +\left.\frac{1}{2} \sum_{n=0}^{N_{V}} \int_{V_{n}} d \vec{r} \int d \vec{r}_{1} d \vec{r}_{2} \frac{\left.\delta^{2} \beta f\left(\vec{r}_{2} ; \Gamma\right]\right)}{\delta \rho\left(\vec{r}_{1}\right) \delta \rho\left(\vec{r}_{2}\right)}\right|_{\rho_{n}} \vec{r}_{1} \vec{r}_{2} \cdot\left(\vec{\nabla} \Gamma_{n, a}\right)\left(\vec{\nabla} \Gamma_{n, b}\right) \frac{\partial \rho\left(\vec{r}_{1} ; \Gamma_{n}\right)}{\partial \Gamma_{n, a}} \frac{\partial \rho\left(\vec{r}_{2} ; \Gamma_{n}\right)}{\partial \Gamma_{n, b}}
\end{aligned}
$$

The second term on the right vanishes by virtue of reflection symmetry in a Bravais lattice, $\rho\left(-\vec{r}_{1} ; \Gamma_{n}\right)=\rho\left(\vec{r}_{1} ; \Gamma_{n}\right)$. The only explicit dependence on $\vec{r}$ is via $\phi\left(\vec{r} ;\left[\rho_{n}\right]\right)$ and the integral of this quantity and its derivatives over $V_{0}$ can 
be extended to an integral over all space and using

$$
\begin{aligned}
\int_{V_{0}} d \vec{r} f\left(\vec{r} ;\left[\rho_{n}\right]\right) & =\frac{1}{N} \int d \vec{r} f\left(\vec{r} ;\left[\rho_{n}\right]\right)=\frac{1}{N} F_{e x}\left[\rho_{n}\right] \\
\left.\int_{V_{n}} d \vec{r} \frac{\delta \beta f(\vec{r} ;[\Gamma])}{\delta \rho\left(\vec{r}_{1}\right)}\right|_{\rho_{n}} & =\left.\frac{1}{N} \frac{\delta \beta F_{e x}[\rho]}{\delta \rho\left(\vec{r}_{1}\right)}\right|_{\rho_{n}}=-\frac{1}{N} c_{1}\left(\vec{r}_{1} ;\left[\rho_{n}\right]\right) \\
\left.\int_{V_{n}} d \vec{r} \frac{\left.\delta^{2} f\left(\vec{r}_{2} ; \Gamma\right]\right)}{\delta \rho\left(\vec{r}_{1}\right) \delta \rho\left(\vec{r}_{2}\right)}\right|_{\rho_{n}} & =\left.\frac{1}{N} \frac{\delta^{2} \beta F_{e x}[\rho]}{\delta \rho\left(\vec{r}_{1}\right) \delta \rho\left(\vec{r}_{2}\right)}\right|_{\rho_{n}}=-\frac{1}{N} c_{2}\left(\vec{r}_{1}, \vec{r}_{2} ;\left[\rho_{n}\right]\right)
\end{aligned}
$$

gives

$$
\begin{aligned}
\beta F[\Gamma]= & \frac{1}{N} \sum_{n=0}^{N_{V}} F_{e x}\left[\rho_{n}\right] \\
& -\frac{1}{2 N} \sum_{n=0}^{N_{V}} \int d \vec{r}_{1} c_{1}\left(\vec{r}_{1} ;\left[\rho_{n}\right]\right) \vec{r}_{1} \vec{r}_{1} \cdot\left(\vec{\nabla} \vec{\nabla} \Gamma_{n, a}\right) \frac{\partial \rho\left(\vec{r}_{1} ; \Gamma_{n}\right)}{\partial \Gamma_{n, a}} \\
& -\frac{1}{2 N} \sum_{n=0}^{N_{V}} \int d \vec{r}_{1} c_{1}\left(\vec{r}_{1} ;\left[\rho_{n}\right]\right) \vec{r}_{1} \vec{r}_{1} \cdot\left(\vec{\nabla} \Gamma_{n, a}\right)\left(\vec{\nabla} \Gamma_{n, b}\right) \frac{\partial^{2} \rho\left(\vec{r}_{1} ; \Gamma_{n}\right)}{\partial \Gamma_{n, a} \partial \Gamma_{n, b}} \\
& -\frac{1}{2 N} \sum_{n=0}^{N_{V}} \int d \vec{r}_{1} d \vec{r}_{2} c_{2}\left(\vec{r}_{1}, \vec{r}_{2} ;\left[\rho_{n}\right]\right) \vec{r}_{1} \vec{r}_{2} \cdot\left(\vec{\nabla} \Gamma_{n, a}\right)\left(\vec{\nabla} \Gamma_{n, b}\right) \frac{\partial \rho\left(\vec{r}_{1} ; \Gamma_{n}\right)}{\partial \Gamma_{n, a}} \frac{\partial \rho\left(\vec{r}_{2} ; \Gamma_{n}\right)}{\partial \Gamma_{n, b}}
\end{aligned}
$$

This represents the desired coarse-graining of the dependence of the order parameter on the microscopic structure. This is made clearer by writing the free energy as

$$
\beta F[\Gamma]=\sum_{n=0}^{N_{V}}\left(\begin{array}{c}
K_{0}\left(\Gamma\left(\vec{R}_{n}\right)\right)+\sum_{a} \overleftrightarrow{K}_{1, a}\left(\Gamma\left(\vec{R}_{n}\right)\right):\left(\vec{\nabla} \vec{\nabla} \Gamma_{a}\right)_{\vec{r}=\vec{R}_{n}} \\
+\sum_{a b} \overleftrightarrow{K}_{2, a b}\left(\Gamma\left(\vec{R}_{n}\right)\right):\left(\vec{\nabla} \Gamma_{a}\right)_{\vec{r}=\vec{R}_{n}}\left(\vec{\nabla} \Gamma_{b}\right)_{\vec{r}=\vec{R}_{n}}
\end{array}\right)
$$

with

$$
\begin{aligned}
K_{0}\left(\Gamma\left(\vec{R}_{n}\right)\right)= & \frac{1}{N} F_{e x}\left[\rho_{n}\right] \\
\overleftrightarrow{K}_{1, a}\left(\Gamma\left(\vec{R}_{n}\right)\right)= & -\frac{1}{2 N} \int d \vec{r}_{1} c_{1}\left(\vec{r}_{1} ;\left[\rho_{n}\right]\right) \vec{r}_{1} \vec{r}_{1} \frac{\partial \rho\left(\vec{r}_{1} ; \Gamma_{n}\right)}{\partial \Gamma_{n, a}} \\
\overleftrightarrow{K}_{2, a b}\left(\Gamma\left(\vec{R}_{n}\right)\right)= & -\frac{1}{2 N} \int d \vec{r}_{1} c_{1}\left(\vec{r}_{1} ;\left[\rho_{n}\right]\right) \vec{r}_{1} \vec{r}_{1} \frac{\partial^{2} \rho\left(\vec{r}_{1} ; \Gamma_{n}\right)}{\partial \Gamma_{n, a} \partial \Gamma_{n, b}} \\
& -\frac{1}{2 N} \int d \vec{r}_{1} d \vec{r}_{2} c_{2}\left(\vec{r}_{1}, \vec{r}_{2} ;\left[\rho_{n}\right]\right) \vec{r}_{1} \vec{r}_{2} \frac{\partial \rho\left(\vec{r}_{1} ; \Gamma_{n}\right)}{\partial \Gamma_{n, a}} \frac{\partial \rho\left(\vec{r}_{2} ; \Gamma_{n}\right)}{\partial \Gamma_{n, b}}
\end{aligned}
$$

\section{B. Resummation}

Having obtained the form given in eq.(46), the question is whether the lattice sums might be written as integrals so as to obtain something like the postulated form given in eq.(31). This question has not been addressed in previous derivations. To do the resummation, we first write the lattice sums more explicitly. Let the primitive lattice vectors for the $D$-dimensional lattice be $\left\{\vec{a}_{i}\right\}_{i=1}^{D}$ so that the set of lattice vectors can be written as $\left\{\vec{R}_{n}\right\}_{n=1}^{n_{V}}=\left\{\sum_{i=1}^{D} m_{i} \vec{a}_{i}\right\}_{m_{1} \ldots m_{D}=M_{1} \ldots M_{D}}^{N_{1} \ldots N_{D}}$ where the various limits, $M_{i}$ and $N_{i}$, will depend on the geometry. Then, for any function of position $f(\vec{r})$ one has that

$$
\sum_{n} f\left(\vec{R}_{n}\right)=\sum_{m_{D}=M_{D}}^{N_{D}} \ldots \sum_{m_{1}=M_{1}\left(m_{2} \ldots m_{D}\right)}^{N_{1}\left(m_{2} \ldots m_{D}\right)} f\left(m_{1} \vec{a}_{1}+\ldots+m_{D} \vec{a}_{D}\right),
$$

where, as indicated, the limits of the inner sums must be allowed to depend on the indices of the outer sums so as to allow for the most general boundary conditions. For simplicity, the dependence of the limits on the indices will 
be suppressed but it should always be considered to be present. This summation can be expressed as an integral by means of the Euler-Maclaurin summation formula giving the exact relation

$$
\begin{aligned}
& \sum_{m_{1}=M_{1}}^{N_{1}} f\left(m_{1} \vec{a}_{1}+\ldots+m_{D} \vec{a}_{D}\right) \\
= & \int_{M_{1}}^{N_{1}}\left[f\left(x \vec{a}_{1}+\ldots+m_{D} \vec{a}_{D}\right)+\frac{(-1)^{r}}{(r+1) !} B_{r+1}(x) \frac{\partial^{r+1}}{\partial x^{r+1}} f\left(x \vec{a}_{1}+\ldots+m_{D} \vec{a}_{D}\right)\right] d x \\
& +\sum_{k=1}^{r} \frac{(-1)^{k+1} B_{k+1}}{(k+1) !}\left[\frac{\partial^{k}}{\partial x^{k}} f\left(x \vec{a}_{1}+\ldots+m_{D} \vec{a}_{D}\right)\right]_{M_{1}}^{N_{1}}
\end{aligned}
$$

where $B_{k}$ is the k-th Bernoulli number, $B_{k}(x)$ is the $\mathrm{k}$-th Bernoulli periodic function and $r$ is an arbitrarily chosen positive integer. The Bernoulli functions are polynomials for $x \in[0,1]$ and are defined outside this interval by $B_{k}(x)=B_{k}(x \bmod 1)$. Thus, they are bounded for all values of $k$ and in fact it is also true that their integral over the unit interval vanishes. The idea here is that since only terms up to order $n_{0}$ are retained, and since the functions $B_{k}(x)$ are bounded, the last term on the right can be neglected provided $r+1>n_{0}$. The second term on the right is evaluated on the boundary. For $r=1$, it vanishes provided

$$
\left.\frac{\partial}{\partial x} f\left(x \vec{a}_{1}+\ldots+m_{D} \vec{a}_{D}\right)\right|_{x=N_{1}}=\left.\frac{\partial}{\partial x} f\left(x \vec{a}_{1}+\ldots+m_{D} \vec{a}_{D}\right)\right|_{x=M_{1}}
$$

which could be true either because of the boundary conditions or because of symmetry. Even if it does not vanish, its contribution will be of order $\frac{1}{L}$ compared to the first term , where $L=\left|\left(N_{1}-M_{1}\right) \vec{a}_{1}\right|$ so that in the thermodynamic limit, it should not contribute. Choosing $r$ to be arbitrarily large and demanding that

$$
\sum_{k=1}^{r} \frac{(-1)^{k+1} B_{k+1}}{(k+1) !}\left[\frac{\partial^{k}}{\partial x^{k}} f\left(x \vec{a}_{1}+\ldots+m_{D} \vec{a}_{D}\right)\right]_{M_{1}}^{N_{1}}=0
$$

imposes a more complex boundary condition. To illustrate, in one dimension, $r=3$ gives

$$
\frac{a_{1}}{12}\left[f^{\prime}\left(N_{1} a_{1}\right)-f^{\prime}\left(M_{1} a_{1}\right)\right]-\frac{a_{1}^{3}}{720}\left[f^{(3)}\left(N_{1} a_{1}\right)-f^{(3)}\left(M_{1} a_{1}\right)\right]=0 .
$$

It could be argued that in the present case, since only terms up to second order in the gradients of the order parameters are kept, the third order terms in this boundary condition could in any case be neglected so that any choice of $r \geq 2$ would be satisfactory. It seems best, however, to note that $B_{3}=0$ so that taking $r=2$ avoids any subtleties that might arise on the boundary. Similarly, if one were keeping gradients up to order $n_{0}$, and $n_{0}$ is even, one would take $r=n_{0}$ with the consequent boundary conditions involving derives up to order $n_{0}-1$. No such simple choice is available if $n_{0}$ is odd.

So, for the present case of a second order theory taking $r=2$ gives, when third derivatives are neglected,

$$
\sum_{m_{1}=M_{1}}^{N_{1}} f\left(m_{1} \vec{a}_{1}+\ldots+m_{D} \vec{a}_{D}\right) \simeq \int_{M_{1}}^{N_{1}} f\left(x \vec{a}_{1}+\ldots+m_{D} \vec{a}_{D}\right) d x
$$

together with the boundary condition, eq.(50). Furthermore, it happens that $\left|B_{3}(x)\right| \leq \frac{1}{36} \sqrt{3} \simeq 5 \times 10^{-2}$ and that $B_{3}(x)$ is oscillatory over the distance of one lattice spacing whereas the order parameters are meant to vary slowly over such small distances, thus further strengthening the argument for neglecting the higher-order term. When this is extended to include the second sum, a new complication arises since this gives

$$
\begin{aligned}
\sum_{m_{2}=M_{2}}^{N_{2}} \sum_{m_{1}=M_{1}}^{N_{1}} f\left(m_{1} \vec{a}_{1}+\ldots+m_{D} \vec{a}_{D}\right) \simeq & \int_{M_{2}}^{N_{2}} d y \int_{M_{1}}^{N_{1}} d x f\left(x \vec{a}_{1}+y \vec{a}_{2}+\ldots+m_{D} \vec{a}_{D}\right) \\
& +\frac{B_{2}}{2}\left[\frac{\partial}{\partial y} \int_{M_{1}}^{N_{1}} d x f\left(x \vec{a}_{1}+y \vec{a}_{2} \ldots+m_{D} \vec{a}_{D}\right)\right]_{M_{2}}^{N_{2}} \\
& +\frac{1}{3 !} \int_{M_{2}}^{N_{2}} d y B_{3}(y) \frac{\partial^{3}}{\partial y^{3}} \int_{M_{1}}^{N_{1}} d x f\left(x \vec{a}_{1}+y \vec{a}_{2}+\ldots+m_{D} \vec{a}_{D}\right)
\end{aligned}
$$


In the event that $M_{1}$ and $N_{1}$ depend on $y$, as they would e.g. for a spherical boundary, then the second term on the right becomes

$$
\begin{aligned}
& \int_{M_{1}}^{N_{1}} d x\left[\frac{\partial}{\partial y} f\left(x \vec{a}_{1}+y \vec{a}_{2} \ldots+m_{D} \vec{a}_{D}\right)\right]_{M_{2}}^{N_{2}} \\
& +\left[\frac{\partial N_{1}(y)}{\partial y} f\left(N_{1} \vec{a}_{1}+y \vec{a}_{2} \ldots+m_{D} \vec{a}_{D}\right)-\frac{\partial M_{1}}{\partial y} f\left(M_{1} \vec{a}_{1}+y \vec{a}_{2} \ldots+m_{D} \vec{a}_{D}\right)\right]_{M_{2}}^{N_{2}} .
\end{aligned}
$$

Now, the first contribution can be required to vanish giving a simple and expected multidimensional generalization of eq. (50) while the second term involves only the value of $f$ on the boundary and geometric functions concerning the definition of the boundary. All of these quantities are fixed in the variational procedure leading from eq.(31) to the Euler-Lagrange equations, eq.(33), so they are of no importance. Thus, with the necessary generalization of the boundary condition, the second term on the right of eq.(54) can be neglected. Finally, the third term on the right in eq. (54) becomes

$$
\begin{aligned}
& \int_{M_{2}}^{N_{2}} d y B_{3}(y)\left[\frac{\partial^{3} N_{1}}{\partial y^{3}} f\left(N_{1} \vec{a}_{1}+y \vec{a}_{2}+\ldots+m_{D} \vec{a}_{D}\right)-\frac{\partial^{3} M_{1}}{\partial y^{3}} f\left(M_{1} \vec{a}_{1}+y \vec{a}_{2}+\ldots+m_{D} \vec{a}_{D}\right)\right] \\
& +3 \int_{M_{2}}^{N_{2}} d y B_{3}(y)\left[\frac{\partial^{2} N_{1}}{\partial y^{2}} \frac{\partial}{\partial y} f\left(N_{1} \vec{a}_{1}+y \vec{a}_{2}+\ldots+m_{D} \vec{a}_{D}\right)-\frac{\partial^{2} M_{1}}{\partial y^{2}} \frac{\partial}{\partial y} f\left(M_{1} \vec{a}_{1}+y \vec{a}_{2}+\ldots+m_{D} \vec{a}_{D}\right)\right] \\
& +3 \int_{M_{2}}^{N_{2}} d y B_{3}(y)\left[\frac{\partial N_{1}}{\partial y} \frac{\partial^{2}}{\partial y^{2}} f\left(N_{1} \vec{a}_{1}+y \vec{a}_{2}+\ldots+m_{D} \vec{a}_{D}\right)-\frac{\partial M_{1}}{\partial y} \frac{\partial^{2}}{\partial y^{2}} f\left(M_{1} \vec{a}_{1}+y \vec{a}_{2}+\ldots+m_{D} \vec{a}_{D}\right)\right] \\
& +\int_{M_{2}}^{N_{2}} d y B_{3}(y) \int_{M_{1}}^{N_{1}} d x \frac{\partial^{3}}{\partial y^{3}} f\left(x \vec{a}_{1}+y \vec{a}_{2}+\ldots+m_{D} \vec{a}_{D}\right) .
\end{aligned}
$$

Here, the first term is of no importance since it involves the value of $f$ on the boundary and the fourth term can be neglected as it is of third order. Note that in the remaining terms, $\frac{\partial}{\partial y} f\left(N_{1} \vec{a}_{1}+y \vec{a}_{2}+\ldots+m_{D} \vec{a}_{D}\right)$ is a derivative evaluated for variations on the boundary, as are all of the other derivatives that occur in the second and third terms. Thus, if the boundary condition is that the order parameters assume constant values on the boundary, then these terms are negligible. This type of boundary condition is certainly reasonable for spherically symmetric problems or on the radial boundary for those with cylindrical symmetry. On the other hand, for planar interfaces one could take the volume to be defined by $N_{i}=-M_{i}=N$ for some constant $N$ in which case $\frac{\partial N_{1}}{\partial y}=0$, etc. and again the second and third contributions are negligible. Again, these terms should be of no consequence in the thermodynamic limit as they are of order $\frac{1}{L}$ compared to the first term in eq.(54). Thus, with these considerations, it has been proven that up to third order gradients,

$$
\sum_{m_{2}=M_{2}}^{N_{2}} \sum_{m_{1}=M_{1}}^{N_{1}} f\left(m_{1} \vec{a}_{1}+\ldots+m_{D} \vec{a}_{D}\right) \simeq \int_{M_{2}}^{N_{2}} d y \int_{M_{1}}^{N_{1}} d x f\left(x \vec{a}_{1}+y \vec{a}_{2}+\ldots+m_{D} \vec{a}_{D}\right) .
$$

Clearly, this result can be extended to any number of dimensions to get the general result

$$
\sum_{m_{D}=M_{D}}^{N_{D}} \ldots \sum_{m_{1}=N_{1}}^{N_{1}} f\left(m_{1} \vec{a}_{1}+\ldots+m_{D} \vec{a}_{D}\right)=\int_{M_{D}}^{N_{D}} \ldots \int_{M_{1}}^{N_{1}} d x_{1} \ldots d x_{D} f\left(x_{1} \vec{a}_{1}+\ldots+x_{D} \vec{a}_{D}\right)
$$

which is expected to hold as the limits go to infinity or, for finite systems, with the corresponding boundary conditions

$$
\forall i,\left.\frac{\partial}{\partial x_{i}} f\left(x_{1} \vec{a}_{1}+\ldots+x_{D} \vec{a}_{D}\right)\right|_{x_{i}=N_{i}}=\left.\frac{\partial}{\partial x_{i}} f\left(x_{1} \vec{a}_{1}+\ldots+x_{D} \vec{a}_{D}\right)\right|_{x_{i}=M_{i}} .
$$

and $f$ is either a constant on the boundaries or the boundaries are defined by fixed values of $M_{i}$ and $N_{i}$. Since the primitive lattice vectors are linearly independent, the integral can be written as a volume integral by a change of variables giving

$$
\sum_{m_{D}=M_{D}}^{N_{D}} \ldots \sum_{m_{1}=N_{1}}^{N_{1}} f\left(m_{1} \vec{a}_{1}+\ldots+m_{D} \vec{a}_{D}\right)=\frac{1}{V_{w s}} \int_{V}\left[f(\vec{r})+O\left(\nabla^{3} f\right)\right] d \vec{r}+\chi
$$

where $\chi$ represents the neglected terms which involve the value of $f$ on the boundaries and the various geometric factors. It is important in terms of calculating the absolute value of the free energy, but plays no role in formulating the Euler-Lagrange equations for the order parameters. 


\section{Continuum Limit}

Making use of eq.(60) and $N V_{w s}=V$, one has that, up to second order in the gradients and an overall additive constant, that

$$
\begin{aligned}
\beta F[\Gamma]= & \frac{1}{V} \int d \vec{R} \beta F[\rho(\Gamma(\vec{R}))] \\
& -\frac{1}{2 V} \int d \vec{r}_{1} d \vec{R} c_{1}\left(\vec{r}_{1} ;[\rho(\Gamma(\vec{R}))]\right) \vec{r}_{1} \vec{r}_{1}: \frac{\partial}{\partial \vec{R}}\left(\frac{\partial \Gamma_{a}(\vec{R})}{\partial \vec{R}\left(\vec{r}_{1} ; \Gamma(\vec{R})\right)} \frac{\partial \Gamma_{a}(\vec{R})}{2 V}\right) \\
& -\frac{1}{2 V} \int d \vec{r}_{1} d \vec{r}_{2} d \vec{R} c_{12}\left(\vec{r}_{1}, \vec{r}_{2} ;[\rho(\Gamma(\vec{R}))]\right)\left[\begin{array}{c}
\vec{r}_{1} \vec{r}_{2}: \frac{\partial \Gamma_{a}(\vec{R})}{\partial \vec{R}} \frac{\partial \Gamma_{b}(\vec{R})}{\partial \vec{R}} \\
\left.\times \frac{\partial \rho\left(\vec{r}_{1} ; \Gamma(\vec{R})\right)}{\partial \Gamma_{a}(\vec{R})} \frac{\partial \rho\left(\vec{r}_{2} ; \Gamma(\vec{R})\right)}{\partial \Gamma_{a}(\vec{R})}\right] .
\end{array}\right.
\end{aligned}
$$

An integration by parts gives

$$
\begin{aligned}
\beta F[\Gamma]= & \frac{1}{V} \int d \vec{R} \beta F[\rho(\Gamma(\vec{R}))] \\
& -\frac{1}{2 V} \int d \vec{r}_{1} d \vec{r}_{2} d \vec{R}\left[\begin{array}{c}
\left.c_{12}\left(\vec{r}_{1}, \vec{r}_{2} ;[\rho(\Gamma(\vec{R}))]\right)\left(\vec{r}_{1} \vec{r}_{2}-\vec{r}_{1} \vec{r}_{1}\right): \frac{\partial \Gamma_{a}(\vec{R})}{\partial \vec{R}} \frac{\partial \Gamma_{b}(\vec{R})}{\partial \vec{R}}\right] \\
\times \frac{\partial \rho\left(\vec{r}_{1} ; \Gamma(\vec{R})\right)}{\partial \Gamma_{a}(\vec{R})} \frac{\partial \rho\left(\vec{r}_{2} ; \Gamma(\vec{R})\right)}{\partial \Gamma_{b}(\vec{R})}
\end{array}\right] \\
& -\frac{1}{2 V} \int d \vec{r}_{1} d \vec{R} \frac{\partial}{\partial \vec{R}} \cdot c_{1}\left(\vec{r}_{1} ;[\rho(\Gamma(\vec{R}))]\right) \vec{r}_{1} \vec{r}_{1} \cdot\left(\frac{\partial \Gamma_{a}(\vec{R})}{\partial \vec{R}_{2}} \frac{\left.\partial \vec{r}_{1} ; \Gamma(\vec{R})\right)}{\partial \Gamma_{a}(\vec{R})}\right)
\end{aligned}
$$

or, rearranging the second term and using Gauss's theorem on the third term,

$$
\begin{aligned}
\beta F[\Gamma]= & \int d \vec{R}\left[\frac{1}{V} \beta F(\Gamma(\vec{R}))+\frac{1}{2} K_{i j}^{a b}(\Gamma(\vec{R})) \frac{\partial \Gamma_{a}(\vec{R})}{\partial R_{i}} \frac{\partial \Gamma_{b}(\vec{R})}{\partial R_{j}}\right] \\
& -\frac{1}{2 V} \int d \vec{r}_{1} \int_{S(V)} c_{1}\left(\vec{r}_{1} ;[\rho(\Gamma(\vec{R}))]\right) \vec{r}_{1} \cdot\left(\frac{\partial \Gamma_{a}(\vec{R})}{\partial \vec{R}} \frac{\partial \rho\left(\vec{r}_{1} ; \Gamma(\vec{R})\right)}{\partial \Gamma_{a}(\vec{R})}\right) \vec{r}_{1} \cdot d \vec{S}
\end{aligned}
$$

with

$$
K_{i j}^{a b}(\Gamma)=\frac{1}{2 V} \int d \vec{r}_{1} d \vec{r}_{2} r_{12 i} r_{12 j} c_{2}\left(\vec{r}_{1}, \vec{r}_{2} ; \rho_{\Gamma}\right) \frac{\partial \rho\left(\vec{r}_{1} ; \Gamma\right)}{\partial \Gamma_{a}} \frac{\partial \rho\left(\vec{r}_{2} ; \Gamma\right)}{\partial \Gamma_{b}}
$$

and where the surface term involves an integral of $\vec{R}$ over the boundary of the integration volume $V, S(V)$. Except for the surface term, this is the desired result. In the thermodynamic limit, the surface term gives negligible contribution, as long as the order parameters and their derivatives are finite on the boundary.

\section{CONCLUSIONS}

In this paper, it has been shown that, up to second order in the gradients of the order parameters and neglecting various constants which depend on the value of the density on the boundaries of the region of interest, the Helmholtz free energy of an arbitrary simple crystalline system with fixed lattice structure can be written in the form of eq.(31) thus generalizing the results of Löwen, et al[5],[6]. This expression makes a cleaner separations of length scales than does the older form, eq.(27). Furthermore, at no point in this derivation were higher order correlations arbitrarily neglected: the only approximation made was to truncate the gradient expansion at second order. This directly addresses some criticisms of the use of these types of models, namely that they are too crude in the treatment of correlations[10]. 
The applicability of eq.(64) depends on the boundary conditions. It requires no qualification in the thermodynamic limit, except that it is assumed that the order parameters and their derivatives are finite on the boundary and the usual derivation of the Euler-Lagrange equations require that the values of the order parameters be constant on the boundary. For finite systems, the resummation of the local free energies requires that

$$
\forall i,\left.\frac{\partial}{\partial x_{i}} \Gamma\left(x_{1} \vec{a}_{1}+\ldots+x_{D} \vec{a}_{D}\right)\right|_{x_{i}=N_{i}}=\left.\frac{\partial}{\partial x_{i}} \Gamma\left(x_{1} \vec{a}_{1}+\ldots+x_{D} \vec{a}_{D}\right)\right|_{x_{i}=M_{i}}
$$

and that $\Gamma$ is either a constant on the boundaries or that $\frac{\partial N_{i}}{\partial x_{j}}=0$, etc.Problems involving spherical or cylindrical symmetry will generally automatically ensure the constancy of $\Gamma$ on the boundary and planar interfaces can be handled by assuming a volume defined by $N_{i}=-M_{i}=N$ for some constant $N$. For finite systems, the surface term in eq.(64) must also be considered.

One noteworthy point about the Ginzburg-Landau form, eq.(64), is that all of the microscopic quantities are evaluated for spatially uniform order parameters or, in other words, for bulk systems. Thus, subject to the approximation inherent in the gradient expansion, this technique provides a framework for using good models of bulk systems to study interfacial systems.

One important problem not addressed here is that of elastic relaxation. In general, one would like to allow the lattice parameter to be included in the list of order parameters so that the lattice can expand or contract as one passes from, say, a bulk solid phase into a bulk liquid phase. In fact, this model has been used for a similar problem in which the lattice is allowed to vary from FCC to BCC[11]. The present derivation is not sufficiently general to allow for this as it would mean allowing the volumes $V_{n}$ to depend on the order parameters. This should be possible but the results can be expected to be considerably more complex than those presented here. Such a generalization will be the subject of a future publication.

\section{Acknowledgments}

This work has benefited from conversations I have had with Marc Baus and Pierre Gaspard. This work was supported in part by the European Space Agency under contract number C90105.

[1] R. Evans, Adv. Phys. 28, 143 (1979).

[2] J.-P. Hansen and I. McDonald, Theory of Simple Liquids (Academic Press, San Diego, Ca, 1986).

[3] A. D. J. Haymet and D. W. Oxtoby, J. Chem. Phys. 74, 2559 (1981).

[4] D. W. Oxtoby and A. D. J. Haymet, J. Chem. Phys. 76, 6262 (1982).

[5] H. Löwen, T. Beier, and H. Wagner, Europhys. Lett. 9, 791 (1989).

[6] H. Löwen, T. Beier, and H. Wagner, Z. Phys. B 79, 109 (1990).

[7] R. Ohnesorge, H. Lowen, and H. Wagner, Phys. Rev. A 43, 2870 (1991).

[8] Y. C. Shen and D. W. Oxtoby, J. Chem. Phys. 104, 4233 (1996).

[9] Y. C. Shen and D. W. Oxtoby, Phys. Rev. Lett. 77, 3585 (1996).

[10] Tarazona, Phys. Rev. E 47, 4284 (1993).

[11] Y. C. Shen and D. W. Oxtoby, Phys. Rev. Lett. 77, 3585 (1996). 